AIDS, Publish Ahead of Print

DOI: 10.1097/QAD.0000000000002764

\title{
Impact of rosuvastatin on atherosclerosis in people with HIV at moderate cardiovascular risk; a randomised, controlled trial.
}

Running Title: Rosuvastatin in HIV

Authors: Janine M TREVILLYAN (PhD) ${ }^{1}$, Anthony DART (MBBS) ${ }^{2}$, Eldho PAUL $(\mathrm{PhD})^{3}$, Matthias CAVASSINI (MD) ${ }^{4}$, Jan FEHR (MD) ${ }^{5,6}$, Cornelia STAEHELIN (MD) ${ }^{7}$, Elizabeth $\mathrm{M} \mathrm{DEWAR}^{2}$ (B.Sc), Jennifer F HOY (MBBS) ${ }^{1}$, Alexandra CALMY $(\mathrm{PhD})^{8}$

Affiliations:

1. Department of Infectious Diseases, Alfred Health and Monash University, Melbourne Australia

2. Department of Cardiology, Alfred Health, Melbourne Australia

3. Biostatistics Consulting Platform, Faculty of Medicine, Nursing and Health Sciences, Monash University, Commercial Rd, Melbourne, Victoria, Australia.

4. Division of Infectious Diseases, University Hospital Lausanne, University of Lausanne, Lausanne, Switzerland.

5. Department of Public \& Global Health, Epidemiology, Biostatistics and Prevention Institute, University of Zurich, Zurich Switzerland

6. Division of Infectious Diseases \& Hospital Epidemiology, University Hospital Zurich, Zurich, Switzerland

7. Inselspital, Bern, Switzerland

8. Division of Infectious Diseases, HIV/AIDS Unit, Geneva University Hospitals, Geneva, Switzerland

Details for Corresponding Author:

Dr Janine Trevillyan

Alfred Hospital, Department of Infectious Diseases

55 Commercial Rd,

Melbourne, Victoria, Australia 3004

Email: Janine.trevillyan@monash.edu

Phone: +610390766076

Fax: +61 0390766557

\section{Sources of Funding}

This work was collaboratively funded by the Faculty of Medicine, Nursing and Health Sciences, Monash University; the National Health and Medical Research Council of Australia; the Swiss Foundation of Cardiology; the Swiss National Foundation and the Boninchi Foundation.

Word count: 2016

Copyright @ 2020 Wolters Kluwer Health, Inc. Unauthorized reproduction of this article is prohibited. 
Abstract word count: $250 / 250$

Tables: 2

Supplementary Data: 2 Figures

\section{ABSTRACT}

People living with HIV-1 (PLHIV) are at increased risk for cardiovascular disease.

Objective: This study aimed to determine if PLHIV would benefit from starting statins at a lower threshold than currently recommended in the general population.

Design: A double-blind multicentre, randomised, placebo-controlled trial was performed.

Methods: Participants $(\mathrm{n}=88)$ with well controlled HIV, at moderate cardiovascular risk (Framingham score of $10-15 \%$ ), and not recommended for statins were recruited from Australia and Switzerland. They were randomised 1:1 to rosuvastatin $(n=44) 20 \mathrm{mg}$ daily, $10 \mathrm{mg}$ if co-administered with ritonavir/cobicistat-boosted antiretroviral therapy, or placebo $(\mathrm{n}=40)$ for 96 weeks. Assessments including fasting blood collection and carotid intima media thickness (CIMT) were performed at baseline, and weeks 48 and 96 . The primary outcome was the change from baseline to week 96 in CIMT. (clinicaltrials.gov:NCT01813357)

Results: Participants were predominantly male (82 (97.6\%)); mean age 54 years (SD 6.0). At 96 weeks there was no difference in the progression of CIMT between the rosuvastatin (mean 0.004mm, SE 0.0036) and placebo $(0.0062 \mathrm{~mm}$, SE 0.0039) arms (p value $=0.684$ ), leading to no difference in CIMT levels between groups at week 96 (rosuvastatin arm, 0.7232mm (SE 0.030); placebo arm 0.7785mm (SE 0.032), $\mathrm{p}=0.075)$.

Adverse events were common $(\mathrm{n}=146)$ and predominantly in the rosuvastatin arm (108 [73.9\%]). Participants on rosuvastatin were more likely to cease study medication due to an adverse event $(7[15.9 \%]$ vs $2[5 \cdot 0 \%], \mathrm{p}=0.011)$.

Conclusions: In PLHIV statins prescribed at a lower threshold than guidelines did not lead to improvements in CIMT but was associated with significant adverse events.

Keywords:

1. HIV

2. Cardiovascular Disease

3. Atherosclerosis

4. Primary prevention

5. Statins 


\section{INTRODUCTION}

People living with HIV-1 (PLHIV) are at increased risk for cardiovascular disease (CVD) [1].

Traditional risk scores may underestimate risk in PLHIV as they do not take into account the contributions of antiretroviral therapy, chronic inflammation and immune activation to cardiovascular risk[2]. Thus, their use to determine the need for primary preventative therapy may lead to under-prescription in PLHIV and hence starting statins at a lower threshold than currently recommended in the general population may be appropriate.

This study aimed to determine the effect of rosuvastatin on progression of atherosclerosis in PLHIV at moderate cardiovascular risk.

\section{METHODS}

A double-blind, randomised, placebo-controlled trial was performed to determine the difference in atherosclerotic progression, estimated by carotid intima media thickness (CIMT), in PLHIV at moderate cardiovascular risk randomised to rosuvastatin or placebo for 96 weeks.

Participants were recruited from the Alfred Hospital, Melbourne, Australia and from four HIV clinics within the Swiss HIV Cohort Network in Geneva, Lausanne, Bern, and Zurich.

The study was approved by the Alfred Ethics committee (\#491-12). Protocol available at DOI $10.26180 / 5 \mathrm{e} 4344844 \mathrm{f} 1 \mathrm{c} 8$. All participants provided written informed consent.

Participants were adults with HIV-1 on antiretroviral therapy with a HIV viral load of $<200$ $\mathrm{c} / \mathrm{ml}$ for $\geq$ six months. All had a Framingham risk score (FRS) of $10-15 \%$. Individuals were excluded if they required a statin according to Australian Guidelines (including diabetics, those with prior CVD or a serum cholesterol $>7.5 \mathrm{mmol} / \mathrm{L}$ ) [3]. Those with carotid artery stenosis or $>50 \%$ occlusion due to plaque were ineligible, as were individuals who were currently, or had within six months, taken lipid lowering therapy, antiplatelets, or had a contraindication to statin use. Individuals with a creatinine clearance $<50 \mathrm{ml} / \mathrm{min}$ or $>$ Childs $\mathrm{B}$ cirrhosis or transaminases three times the upper limit of normal were also excluded.

Participants were randomised 1:1 to oral rosuvastatin (20mg daily) or matched placebo, using block randomisation, stratified by country, with a variable block size (range 2-6). The sequence was generated by an independent statistician and conveyed to study pharmacists who had no direct participant contact, all other study team members and participants were blinded. Participants who were taking ritonavir or cobicistat received dose reduced rosuvastatin $(10 \mathrm{mg})$. The rosuvastatin was encapsulated in a gelatin capsule (blue for the $20 \mathrm{mg}$ and red for the $10 \mathrm{mg}$ ) with identical blue and red placebos created by encapsulating sucrose within the same capsules.

Participant demographics and medical history were obtained through self-report and a review of medical records. The FRS was calculated using an online calculator (https://www.mdcalc.com/framingham-risk-score-hard-coronary-heart-disease). 
Participants were evaluated at baseline, weeks $12,24,48,72$, and 96. Laboratory assessments were performed including full blood count, liver function, renal function, electrolytes, creatinine kinase, along with HIV viral load and CD4+ T cell count were measured at each visit, with inflammatory markers (interleukin 6 (IL-6) and high sensitivity c-reactive protein (hsCRP) measured at baseline and week 96. Fasting blood collection were performed following an overnight fast at screening, baseline, week 24, 48 and 96 for lipid and glucose levels.

CIMT was recorded at week 0, 48 and 96. 2D (B-Mode) high resolution ultrasound digital images were obtained at the common carotid artery (CCA), internal carotid artery (ICA) and carotid bulb on both sides. Images were sent to a core laboratory who read all scans in parallel, blinded to treatment allocation, using semi-automated edge detection software (Carotid Analyser, Medical Imaging Applications LLC, Iowa USA). The reported CIMT is the average of the two sides. The CIMT was measured at the end of diastole as determined by simultaneous ECG recordings with measurements made $0-1 \mathrm{~cm}$ from the carotid bulb in $1 \mathrm{~cm}$ length segments from the far wall of the CCA..

The primary outcome measure was the change from baseline to week 96 in CIMT.

Secondary outcomes measures included; the incidence of adverse events, the change from baseline to week 48 in CIMT, and the change in lipid levels at week 48 and 96.

A Data Safety and Monitoring Board (DSMB) reviewed an interim analysis (blinded to allocation) at week 48 . The predefined criteria for early cessation included a significant adverse event rate or statistically significant difference in CIMT progression of $>0.16 \mathrm{~mm}$ between arms, neither of which was met.

Statistical Analysis:

Assuming a CIMT progression rate of $0.080 \mathrm{~mm}$ over 96 weeks in the placebo arm and near stabilisation of CIMT ( $95 \%$ relative reduction) with rosuvastatin, we assumed an effect size of $0.076 \mathrm{~mm}$ (SD $0.09 \mathrm{~mm}$ ) [4]. With an alpha threshold of $1 \%$ and a power of $90 \%$, and $15 \%$ lost-to follow-up over 96 weeks, an initial total study population of 102 was targeted. This was reduced to 84 individuals with the support of the DSMB in August 2016 due to slow recruitment. With 84 participants the study had $90 \%$ power to detect a difference of $0.076 \mathrm{~mm}$ between treatment arms at week 96 (alpha 5\%).

The intention to treat population was as all individuals who received a dose of study medication. The per-protocol set was participants without a major protocol deviation, defined as; the absence of any CIMT assessment, participants on placebo who received any doses of rosuvastatin and participants in the rosuvastatin arm who ceased rosuvastatin or transitioned onto open labelled statin.

The primary outcome was assessed using linear mixed models fitted via restricted maximum likelihood (REML) method with fixed effects for treatment allocation, time of assessment (baseline, 48, 96 weeks), the carotid artery site and body side and their two-way and threeway interactions, along with random effects for study site. These were repeated following adjustment for baseline cardiovascular risk (age, gender, smoking status and LDL). Baseline characteristics were summarised using means (standard deviations (SD)) for continuous 
variables and number (\%) for categorical variables. No adjustment was made for multiple comparisons. Participants with missing data were treated as missing at random and all observed data was considered for analysis with the mixed-effects models assuming noninformative dropout. Statistical significance $\mathrm{p}=0.05$. All analyses were performed using SAS software version 9.4 (SAS Institute, Cary, NC, USA). Figures were drawn using Graph Pad Prism 8.2.1 (2019 San Diego USA).

Clinicaltrials.gov (NCT01813357).

\section{RESULTS:}

Recruitment occurred from July 2013 - August 2016.; follow-up completed May 2018. See supplementary Figure 1, http://links.lww.com/QAD/B916: Participant flowchart.

Participants were predominantly male (97.6\%), 54.1 years (range 42-68) and Caucasian (88.1\%) (see Table 1).

There was no difference in the change in CIMT between the rosuvastatin $(0.004 \mathrm{~mm}$, SE: $0.0036)$ and placebo $(0.0062 \mathrm{~mm}$, SE 0.0039$)$ arms $(\mathrm{p}=0.684)$ at 96 weeks, leading to no difference in CIMT levels between groups (rosuvastatin 0.7232mm (SE 0.030); placebo $0.7785 \mathrm{~mm} \quad$ (SE 0.032), $\mathrm{p}=0.0749$ ) (see supplementary figure 2, http://links.lww.com/QAD/B917). This result remained consistent following adjustment for age, gender, smoking status and LDL cholesterol (delta CIMT from baseline to week 96 rosuvastatin $0.004 \mathrm{~mm}$ (SE 0.0036); placebo $0.0061 \mathrm{~mm}$ (SE 0.0039), $\mathrm{p}=0.694$ ). No difference was detected when the results were restricted to the per-protocol population; delta CIMT baseline to week 96; rosuvastatin $0.0037 \mathrm{~mm}$ (SE 0.004); placebo $0.0066 \mathrm{~mm}$ (SE $0.0042), p=0.616$. No participant had new plaque development in either arm. There was no difference in progression when individual sites of CIMT were compared (see supplementary table 1, http://links.lww.com/QAD/B918).

Rosuvastatin led to predictable reductions in total and LDL-cholesterol by week 24 which was sustained to week 96 . At week 96 mean total cholesterol; rosuvastatin $4.13 \mathrm{mmol} / \mathrm{L}$ (SE 0.156); placebo $5.32 \mathrm{mmol} / \mathrm{L}$ (SE 0.163) $\mathrm{p}<0.001$; LDL-cholesterol; rosuvastatin $2.29 \mathrm{mmol} / \mathrm{L}$ (SE 0.137), placebo $3.35 \mathrm{mmol} / \mathrm{L}$ (SE 0.143), $\mathrm{p}<0.001$. There was no change in HDL cholesterol in either arm from baseline to week 96 (rosuvastatin $1.18 \mathrm{mmol} / \mathrm{L}$ (SE 0.043), placebo $1.23 \mathrm{mmol} / \mathrm{L}$ (SE 0.045), $\mathrm{p}=0.424)$. Neither the baseline total or LDL-cholesterol levels, nor the change from baseline to week 96 in total or LDL-cholesterol, predicted change in CIMT.

There was no change in interleukin-6 (IL-6) levels in either arm (mean change rosuvastatin $0.25 \mathrm{pg} / \mathrm{ml}$ (SD 2.0), placebo $0.03 \mathrm{pg} / \mathrm{ml}$ (SD 1.5), $\mathrm{p}=0.925$ ). Rosuvastatin was associated with decreased hsCRP (mean change rosuvastatin $-0.75 \mathrm{mg} / \mathrm{L}$ (SD 3.8), placebo $1.3 \mathrm{mg} / \mathrm{L}$ (SD 6.6), $\mathrm{p}=0.008$ ).

Fifty-seven (67\%) participants experienced 146 adverse events. Most mild, grade one or two (131 [89.7 (see table 2). These were more common with rosuvastatin (108 [73.9\%]). Participants were more likely to cease study medication due to an adverse event on rosuvastatin $(7[15.9 \%]$ versus $2[5.0 \%])$. There was one death (rosuvastatin arm) from a haemorrhagic cerebrovascular infarct, unrelated to study participation. 


\section{Discussion:}

This study demonstrated no effect of rosuvastatin on progression of CIMT in PLHIV at moderate cardiovascular risk, but rosuvastatin was poorly tolerated with over $15 \%$ of participants interrupting study treatment. This is contrary to previous reports that rosuvastatin was associated with $0.019 \mathrm{~mm}$ less progression of CIMT over 96 weeks [5]. These discordant findings may be explained by baseline differences in enrolled participants, with that study requiring participants to have elevated inflammatory markers at baseline, while our population had low-normal inflammatory marker levels. This would be consistent with the findings from the JUPITER trial which demonstrated a significant reduction in major cardiovascular events in individuals without dyslipidaemia (or HIV) but with an elevated hsCRP

[6]. Suggesting that perhaps utilisation of markers of inflammation, rather than a cardiovascular risk score, may more accurately identify individuals who will benefit from statins.

The CIMT progression of $0.006 \mathrm{~mm}$ in our placebo arm lower than anticipated. This suggests that despite attempting to enrich our study population with those most likely to benefit from therapy participants were in general quite healthy. Perhaps reflecting global trends of reducing CVD in settings in which PLHIV have access to minimally toxic antiretrovirals and durable viral suppression is the norm [7]. Previous observational studies have demonstrated CIMT progression rates of $0.074 \mathrm{~mm}$ (SD $0.13 \mathrm{~mm}$ ) over 1 year in PLHIV which is significantly greater than that seen in the HIV negative controls [8]. Our observed rates are closer to what is described in the general population [9].

CIMT is a well validated surrogate marker of atherosclerosis and a strong predictor of future vascular events [9]. Multiple trials of lipid lowering and antihypertensive interventions have previously used it as an endpoint [10]. While recent meta-analyses found that increased CIMT was associated with future cardiovascular events [11], there have been conflicting results as to whether change in CIMT accurately reflects change in cardiovascular risk.

We had hypothesised that statins, through their pleiotropic anti-inflammatory and immunemodulatory effects [12], may be more effective in PLHIV as HIV is associated with an elevated inflammatory state which persists despite long term viral suppression [13]. Our findings that statin therapy did not lead to significant differences in CIMT and had only minimal effects on inflammatory markers is supported by findings from other groups of minimal effects of statins on biomarkers of HIV persistence, immune activation and inflammation in PLHIV [14]. This raises the possibility that statins anti-inflammatory effects are in pathways less affected by HIV.

An important finding was the high rate of adverse events in those receiving rosuvastatin. While the difference in the mild adverse events is unlikely to be clinically meaningful the four treatment related serious adverse events (two new diagnoses of diabetes and a case each of significant elevations in liver function tests and creatinine kinase) are very important when considering starting a statin in a PLHIV in whom the benefit is not yet clear. There are a number of drug interactions between antiretrovirals and statins, which can lead to elevated side effect risk. To minimise this, participants receiving ritonavir or cobicistat were prescribed dose reduced $(10 \mathrm{mg})$ rosuvastatin. As such we do not believe that drug interactions are responsible for the high rates of side effects observed. 
This study has a number of limitations, notably its small sample size and homogenous participant population. It is, however strengthened by its multinational recruitment and robust randomised placebo-controlled design. While CIMT is a well validated surrogate endpoint for cardiovascular events [15], it may not truly reflect the impact of rosuvastatin on hard coronary endpoints and perhaps the choice of an alternate surrogate endpoint may have resulted in a different outcome. We thus await with interest the results of the Randomized Trial to Prevent Vascular Events in HIV (REPRIEVE) Trial [16], a prospective, randomised placebo controlled trial of pitavastatin for the primary prevention of major adverse cardiovascular events in PLHIV.

Conclusions:

In PLHIV at moderate risk rosuvastatin did not alter progression of atherosclerosis but was associated with increased sided effects.

\section{Acknowledgments:}

The authors would like to acknowledge Ms Kerrie Watson for assistance with study database design, data entry and cleaning of data base and Dr John Reynolds for independent statistical support. We are also very grateful to the members of the DSMB, Ptof Andrew Carr (chair), Dr Peter Bergin, Dr Giovanni Guaraldi, Dr Christoph Meier, Dr Kathy Petoumenos.

\section{Sources of Funding}

This work was collaboratively funded by the Faculty of Medicine, Nursing and Health Sciences, Monash University; the National Health and Medical Research Council of Australia; the Swiss Foundation of Cardiology; the Swiss National Foundation and the Boninchi Foundation.

\section{Conflicts of interest}

Dr Trevillyan has received honoraria from Gilead Health Sciences for speaker responsibilities unrelated to this project. Professor Hoy's institution has received reimbursement for her participation in Advisory Boards for Gilead Sciences, ViiV Healthcare and MSD. Professor Calmy's institution has received unrestricted educational grants from Gilead Health Sciences, ViiV, AbbVie and MSD.

\section{Authors' Contributions:}

JMT: Wrote study protocol, recruited and monitored Australian participants,co-ordinated trial completion, assisted with collation and analysis of data, wrote manuscript.

AD: Provided expert cardiology advice on study design and interpretation of results

EP: Development of the statistical analysis plan and analysis of results

MC: co-ordinated trial in Lausanne

JF: Co-ordinated trial in Zurich

CS: Co-ordinated trial in Berne

EMD: Developed CIMT standard operating procedure and performed scanning in Australia

JFH: Conceived study idea, contributed to protocol development, supervised co-ordination of the trial in Australia, contributed to analysis of results and drafting of manuscript

AC: Supervised co-ordination of the trial in Switzerland, contributed to protocol development and drafting of the manuscript 


\section{REFERENCES:}

1. Paisible AL, Chang CC, So-Armah KA, Butt AA, Leaf DA, Budoff M, ... Freiberg MS. HIV Infection, Cardiovascular Disease Risk Factor Profile, and Risk for Acute Myocardial Infarction. J Acquir Immune Defic Syndr 2015; 68(2):209-216.

2. Feinstein MJ, Nance RM, Drozd DR, Ning H, Delaney JA, Heckbert SR, . . Crane HM. Assessing and Refining Myocardial Infarction Risk Estimation Among Patients With Human Immunodeficiency Virus: A Study by the Centers for AIDS Research Network of Integrated Clinical Systems. JAMA Cardiol 2017; 2(2):155-162.

3. The National Vascular Disease Prevention Alliance (NVDPA). Australian absolute cardiovascular disease risk calculator. In; 2012.

4. Bots ML, Palmer MK, Dogan S, Plantinga Y, Raichlen JS, Evans GW, . . Group MS. Intensive lipid lowering may reduce progression of carotid atherosclerosis within 12 months of treatment: the METEOR study. Journal of Internal Medicine 2009; 265(6):698707.

5. Longenecker CT, Sattar A, Gilkeson R, McComsey GA. Rosuvastatin slows progression of subclinical atherosclerosis in patients with treated HIV infection. Aids 2016; 30(14):2195-2203.

6. Ridker PM, Danielson E, Fonseca FA, Genest J, Gotto AM, Jr., Kastelein JJ, . . Glynn RJ. Rosuvastatin to prevent vascular events in men and women with elevated $\mathrm{C}$-reactive protein. $N$ Engl J Med 2008; 359(21):2195-2207.

7. Masia M, Padilla S, Garcia JA, Bernardino JI, Campins AA, Asensi V, . . Cohort of HIVAotARNs. Decreasing rates of acute myocardial infarction in people living with HIV: a nationwide cohort study in Spain, 2004-2015. HIV Med 2018.

8. Hsue PY, Lo JC, Franklin A, Bolger AF, Martin JN, Deeks SG, Waters DD. Progression of atherosclerosis as assessed by carotid intima-media thickness in patients with HIV infection. Circulation 2004; 109(13):1603-1608.

9. Lorenz MW, von Kegler S, Steinmetz H, Markus HS, Sitzer M. Carotid intima-media thickening indicates a higher vascular risk across a wide age range: prospective data from the Carotid Atherosclerosis Progression Study (CAPS). Stroke; a journal of cerebral circulation 2006; 37(1):87-92.

10. Nezu T, Hosomi N, Aoki S, Matsumoto M. Carotid Intima-Media Thickness for Atherosclerosis. J Atheroscler Thromb 2016; 23(1):18-31.

11. Den Ruijter HM, Peters SA, Anderson TJ, Britton AR, Dekker JM, Eijkemans MJ, ... Bots ML. Common carotid intima-media thickness measurements in cardiovascular risk prediction: a meta-analysis. Jama 2012; 308(8):796-803.

12. Montecucco F, Mach F. Update on statin-mediated anti-inflammatory activities in atherosclerosis. Semin Immunopathol 2009; 31(1):127-142.

13. Deeks SG, Lewin SR, Havlir DV. The end of AIDS: HIV infection as a chronic disease. Lancet 2013; 382(9903):1525-1533.

Copyright @ 2020 Wolters Kluwer Health, Inc. Unauthorized reproduction of this article is prohibited. 
14. Bedimo RJ, Mar H, Bosch RJ, Drechsler H, Cyktor JC, Macatangay BJC, . . Gandhi RT. Brief Report: No Evidence for an Association Between Statin Use and Lower Biomarkers of HIV Persistence or Immune Activation/Inflammation During Effective ART. J Acquir Immune Defic Syndr 2019; 82(2):e27-e31.

15. Lorenz MW, Markus HS, Bots ML, Rosvall M, Sitzer M. Prediction of clinical cardiovascular events with carotid intima-media thickness: a systematic review and meta-analysis. Circulation 2007; 115(4):459-467.

16. Grinspoon SK, Fitch KV, Overton ET, Fichtenbaum CJ, Zanni MV, Aberg JA, ... Douglas PS. Rationale and design of the Randomized Trial to Prevent Vascular Events in HIV (REPRIEVE). Am Heart $J$ 2019; 212:23-35. 


\begin{tabular}{|c|c|c|}
\hline & Rosuvastatin & Placebo \\
\hline Age, years & $53.9(5.9)$ & $54.4(6.4)$ \\
\hline Male & $42(95.5 \%)$ & $40(100 \%)$ \\
\hline \multicolumn{3}{|l|}{ Recruitment Site } \\
\hline Melbourne & $28(63.6 \%)$ & $27(67.5 \%)$ \\
\hline Geneva & $4(9.1 \%)$ & $7(17.5 \%)$ \\
\hline Zurich & $8(18.2 \%)$ & $4(10 \%)$ \\
\hline Berne & $3(6.8 \%)$ & $1(2.5 \%)$ \\
\hline Lausanne & $1(2.3 \%)$ & $1(2.5 \%)$ \\
\hline \multicolumn{3}{|l|}{ Race } \\
\hline Caucasian & $40(90.9 \%)$ & $35(81.4 \%)$ \\
\hline Asian & $3(6.8 \%)$ & $2(4.5 \%)$ \\
\hline African & $1(2.2 \%)$ & $6(13.9 \%)$ \\
\hline FRS, $\%$ & $10(1.9)$ & $10(2.0)$ \\
\hline Current smoker & $16(36.4 \%)$ & $12(30 \%)$ \\
\hline Ex-smoker & $15(34.1 \%)$ & $14(35 \%)$ \\
\hline Family History of $\mathrm{AMI}^{\mathrm{a}}$ & $14(31.8 \%)$ & $12(30 \%)$ \\
\hline BMI, $\mathrm{kg} / \mathrm{m}^{2}$ & $26.4(3.6)$ & $26.4(3.3)$ \\
\hline $\mathrm{SBP}, \mathrm{mmHg}$ & $128(13)$ & $128(14)$ \\
\hline DBP, $\mathrm{mmHg}$ & $85(13)$ & $83(9)$ \\
\hline hsCRP, ml/L & $3.4(3.4)$ & $4.3(6.1)$ \\
\hline IL-6, pg/ml & $2.1(1.8)$ & $2.4(2.9)$ \\
\hline \multicolumn{3}{|l|}{ Lipid Parameters } \\
\hline Total Cholesterol, $\mathrm{mmol} / \mathrm{L}$ & $5.4(0.8)$ & $5.3(1.1)$ \\
\hline LDL-cholesterol, $\mathrm{mmol} / \mathrm{L}$ & $3.5(0.7)$ & $3.5(0.9)$ \\
\hline HDL-cholesterol, mmol/L & $1.1(0.3)$ & $1.2(0.3)$ \\
\hline Triglycerides, $\mathrm{mmol} / \mathrm{L}$ & $1.9(0.9)$ & $1.5(0.7)$ \\
\hline Glucose, $\mathrm{mmol} / \mathrm{L}$ & $5.2(0.5)$ & $5(0.5)$ \\
\hline \multicolumn{3}{|l|}{ HIV specific Variables } \\
\hline Time from HIV diagnosis, years & $17.2(8.5)$ & $13.6(7.6)$ \\
\hline Current CD4 cell count, cells/ul & $693(259)$ & $550(254)$ \\
\hline Nadir CD4 cell count, cells/ul & 217 (169) & $159(137)$ \\
\hline Undetectable viral load ${ }^{\mathrm{b}}$ & $44(100 \%)$ & $40(100 \%)$ \\
\hline \multicolumn{3}{|l|}{ Current Antiretroviral therapy } \\
\hline Protease inhibitor & $18(41.9 \%)$ & $18(45 \%)$ \\
\hline NNRTI & $19(44.2 \%)$ & $21(52.5 \%)$ \\
\hline Integrase inhibitor & $12(27.9 \%)$ & $13(32.5 \%)$ \\
\hline Abacavir & $10(23.3 \%)$ & $10(25 \%)$ \\
\hline Tenofovir $^{\mathrm{c}}$ & $28(65.1 \%)$ & $25(62.5 \%)$ \\
\hline
\end{tabular}

Mean (standard deviation) or $\mathrm{n}(\%)$ as appropriate

${ }^{a}$ Defined as a history of AMI in a male first degree relative before 55 years of age, or a female first degree relative before 65 years of age

${ }^{\mathrm{b}}$ Defined as $<200$ copies $/ \mathrm{ml}$

${ }^{\mathrm{c}}$ All tenofovir disaproxyl fumerate except for two tenofovir alafenamide (one in each arm)

Abbreviations: Acute Myocardial Infarction (AMI); Body Mass Index (BMI); Diastolic Blood Pressure (DBP); Framingham Risk Score (FRS); High-density Lipoprotein (HDL); High sensitivity C-reactive protein (hsCRP); Interleukin- 6 (IL-6); Low-density Lipoprotein (LDL); Non-nucleoside Reverse Transcriptase Inhibitors (NNRTI); Systolic Blood Pressure (SBP) 
Table 2: Summary of Adverse Events

\begin{tabular}{|c|c|c|}
\hline & Rosuvastatin & Placebo \\
\hline \multirow[t]{2}{*}{ Any Adverse Event } & $35(79.5)$ & $22(55.0)$ \\
\hline & (108 events) & (38 events) \\
\hline \multicolumn{3}{|l|}{ Grade 3 or 4 adverse event ${ }^{a}$} \\
\hline Myocardial Infarction & $2(4.5)$ & $1(2.5)$ \\
\hline Diabetes & $2(4.5)$ & $0(0.0)$ \\
\hline Elevated ALT • 5x ULN & $1(2.2)$ & $1(2.5)$ \\
\hline Elevated Creatine Kinase $\cdot 10 \mathrm{x}$ ULN & $1(2.2)$ & $0(0.0)$ \\
\hline Study drug related serious adverse event ${ }^{\mathrm{b}}$ & $3(6.8)$ & \\
\hline Adverse event leading to study drug discontinuation ${ }^{c}$ & $7(15.9)$ & $2(5)$ \\
\hline \multicolumn{3}{|l|}{ Adverse event $(\cdot 5 \%$ participants) } \\
\hline Elevated Liver enzymes & $13(29.5)$ & $4(10)$ \\
\hline Myopathic Symptoms $^{\mathrm{d}}$ & $1(2.2)$ & $7(17.5)$ \\
\hline Elevated creatinine kinase & & $3(7.5)$ \\
\hline Hypertension & $13(29.5)$ & $11(27.5)$ \\
\hline Gastrointestinal Symptoms & $5(11.3)$ & $4(10)$ \\
\hline
\end{tabular}

\section{Table 2: Footnote}

$\mathrm{n}(\%)$

${ }^{a}$ Additionally, in the rosuvastatin arm there was one incident each of heart failure, hypertension (systolic blood pressure $180 \mathrm{mmHg}$ ) and cerebrovascular disease. In the placebo arm there was one incident each of acute mesenteric ischemia, oesophageal malignancy, lumbar vertebral disc herniation, and haemoptysis.

${ }^{\mathrm{b}}$ probable or possible

${ }^{c}$ Four participants in the rosuvastatin arm stopped study medication because it was recommended they commence open label statin for new onset cardiovascular disease (acute myocardial infarction or cerebrovascular event) or type two diabetes. In thr rousvawtatin arm gastrointestinal intolerances, musculoskeletal pain and elevated creatinine kinase in one instance each led to study drug cessation. In the placebo arm one participant ceased study medication because of ALT rise $>5 x$ upper limit of normal, and one for mild gastrointestinal symptoms.

${ }^{\mathrm{d}}$ Including myalgia and perceived muscle weakness

Abbreviations: Alanine aminotransferase (ALT); Upper limit of normal (ULN) 